\title{
A NEW ECOLOGICAL PRIMARY FLIGHT DISPLAY CONCEPT
}

\author{
Tony Lambregts, FAA, Seattle, WA \\ Richard Rademaker and Erik Theunissen, Delft University of Technology, Delft, The Netherlands
}

\begin{abstract}
In the current generation electronic primary flight displays, all variables are scaled and displayed without regard to the inherent relationship between airplane flight path and speed related variables, and without regard how the controls of thrust and elevator should be used. This leaves the pilot to solely rely on his experience and skills to realize the desired state of aircraft speed and flight path. The goal of the ecological Energy Management Primary Flight Display, is to make constraints and complex relationships between various flight dynamics display variables visible and directly actionable to the pilot. In this context, the energy domain is identified as a means to normalize and visualize the relationships between speed and altitude targets, acceleration, vertical speed and flight path angle and to bring out control guidance cues for the efficient use of elevator and throttle control. The feasibility of such an ecological primary flight display is addressed through an analysis of the scaling dependencies. Following an overview of the design issues and required design decisions, the practicality is addressed through an example implementation and a comparison with existing display formats and design recommendations. Finally, recommendations for research are provided to assess the suitability and effectiveness of such a display in improving pilot control performance and workload.
\end{abstract}

\section{Introduction}

The introduction of the Electronic Flight Instrument System (EFIS) enabled the integration of instruments that show the primary airplane states needed for control on a single display. The transition from multiple electromechanical instruments to a combined depiction on a single electronic display provided the designers both with challenges and opportunities. Konicke [1] addresses both in the description of the design of the Primary Flight Display (PFD) for the Boeing 747-400. In
[2] recommendations for the design of the instruments of the PFD are provided. FAA electronic display design/certification guidance is found in [3].

The separate instruments on today's PFD are not designed to show how the change in one particular airplane state quantitatively influences the other states. An important purpose of the PFD is to provide the pilot with a display that enables him to close the control loop on key airplane variables. Because the current displays provide these variables on independent display scales, it is natural for the pilot to use a Single-Input/Single-Output (SISO) control strategy, most often controlling airspeed with thrust and the vertical flight path with the elevator/pitch control. Use of one control affects not only the target variable, but also the airplane state the pilot intends to control with the other control means. Over time, the pilot builds up a mental model of the airplane dynamic response to each of the control inputs, enabling him to more properly and effectively mix the controls in a way that results in the desired response of a first target variable, without driving the second control variable too far away form is target value. In effect the more experienced a pilot becomes the more he will be able to use an effective mixed "MultiInput/Multi-Output" (MIMO) control strategy. Thus, it is reasonable to expect that, if the PFD can be rearranged to directly indicate how the pilot can best use both controls simultaneously to satisfy the flight path and speed control objectives, the pilots will quicker acquire the proper MIMO control skills to achieve better/smoother performance with less workload/control effort and better awareness of the overall airplane dynamics.

The goal of ecological interface design is to make constraints and complex relationships in the work environment perceptually evident (e.g. visible, audible) to the user. Long before the term ecological interface design (EID) was coined, the concept was already applied in the design of aircraft guidance displays. A nice example is the

U.S. Government work not protected by U.S. Copyright. 
analysis of the task domain performed by Klopfstein [4]. In order not to come-up with a concept that is influenced by the existing instrumentation at the time, Klopfstein starts his analysis in the following way: 'Suppose for a moment that there were no birds or flying insects in our world and that the idea of heavier than air flying machines was a recent one. What would a team of engineers who had just discovered aerodynamic lift and were designing the first flying machine do to make the machine controllable by an airman?'. His answer to this question is comparable to the top-down analysis underlying EID: 'before embarking on their research they would try to define the objectives. The principle aim in piloting a flying machine is of course to take it where the airman wishes to go'. Klopfstein continues with 'in the light of this definition the airman must be given the means of action and the data necessary to accomplish his work'. Based on an analysis of the process, its limitations, the means of control, safety aspects and the properties of sensors that can provide the data, Klopstein illustrates how the explicit presentation of flight path is of fundamental importance to the task. In his study, he relates the concept of total energy and energy-rate to the "total slope" quantity, defined as the sum of airplane Flight Path Angle (FPA) and normalized acceleration. He provides a design rationale for using this "total slope" as a thrust setting display, and using FPA indication in lieu of pitch attitude to close the elevator control loop.

In this paper, the concept of "total slope", here further referred to as Potential Flight Path Angle (PFPA), together with FPA and acceleration along the flight path, are further developed to design PFD instruments wherein the relative airplane energy relationships are made visible and directly usable by the pilot as control cues. The goal is to expand the ideas of Klopfstein to provide the pilot with better awareness of airplane energy-based performance capability and MIMO control cuing information for the efficient application of both thrust and elevator control. A further objective is to provide a generalized "Energy Management" PFD that can support all airplane control modes of operation, including pilot loop closure during manual control and monitoring of automatic control, allowing the entire longitudinal flight control design to be harmonized using a common energy-based control strategy.

\section{Conventional SISO Control}

As for manual control, most automatic flight control system algorithms in use today are of the SISO variety. This is a result of the historic axis by axis one-mode-at-a-time evolution of these systems. As an illustration, Figure 1 presents a conventional SISO flight control system for the capture and tracking of speed and altitude targets. The altitude error is processed to develop the elevator command. The speed error is processed to develop the throttle command.

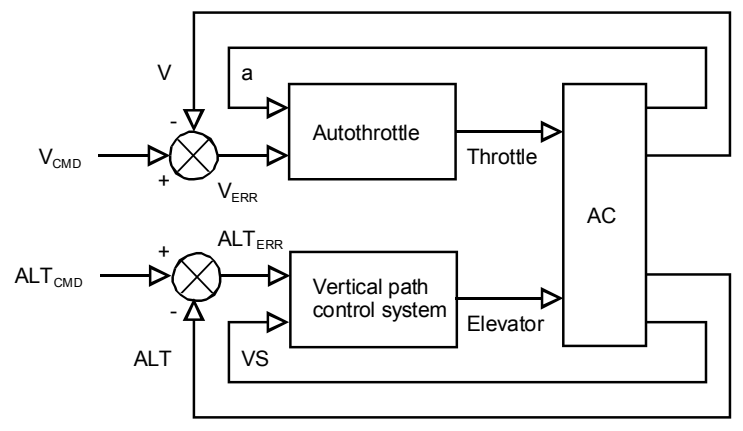

\section{Figure 1. SISO Loops for Altitude and Speed Control}

Typically, there are no feed-forward signal paths from the flight path error to the throttles and from the airspeed error to the elevator to decouple the control responses. Unfortunately, besides the problems of undesirable control coupling and sometimes less than desirable performance, there are a number of situations where this strategy cannot fulfill the functional intention of automation. In these situations, the flight crew must step in to prevent an unsafe condition from developing. This has lead to many flight guidance and control automation safety, performance and design/ operational complexity problems that are difficult to solve in a fully satisfactory and effective manner with the traditional SISO design structure.

A typical SISO control strategy related problem is clearly illustrated by a situation involving a substantial change in the altitude command using elevator control. Besides the desired flight path response, an airspeed error is induced which subsequently drives the throttles to a limit position, after which speed control is lost. 
Speed deviation can then become unbounded, leading to loss of airplane control. To prevent loss of airspeed control in this situation requires some form of speed envelope protection. A workable but complex, and still not ideal solution for his problem, was the development of the Flight Level Change (FLCH) mode in the early eighties. For large altitude changes this mode uses the elevator to control speed during the climb and the throttle to control vertical speed [5]. Still, it is difficult to achieve smooth transitions between the conventional and reversed control strategies for all possible conditions.

The coupling effect of a SISO control strategy for vertical path and speed control can be visualized by plotting the energy state of an aircraft during the transition to a new target state. The energy state of an aircraft can be described by its potential energy $E_{P}$ and its kinetic energy $E_{K}$. This state can be visualized in an energy graph by plotting $E_{k}$ on the $\mathrm{x}$-axis and $\mathrm{E}_{\mathrm{p}}$ on the $\mathrm{y}$-axis. The behavior of a typical SISO "Speed on Throttle" and "Flight Path on Elevator" automatic control loops is illustrated in an energy graph of Figure 2.

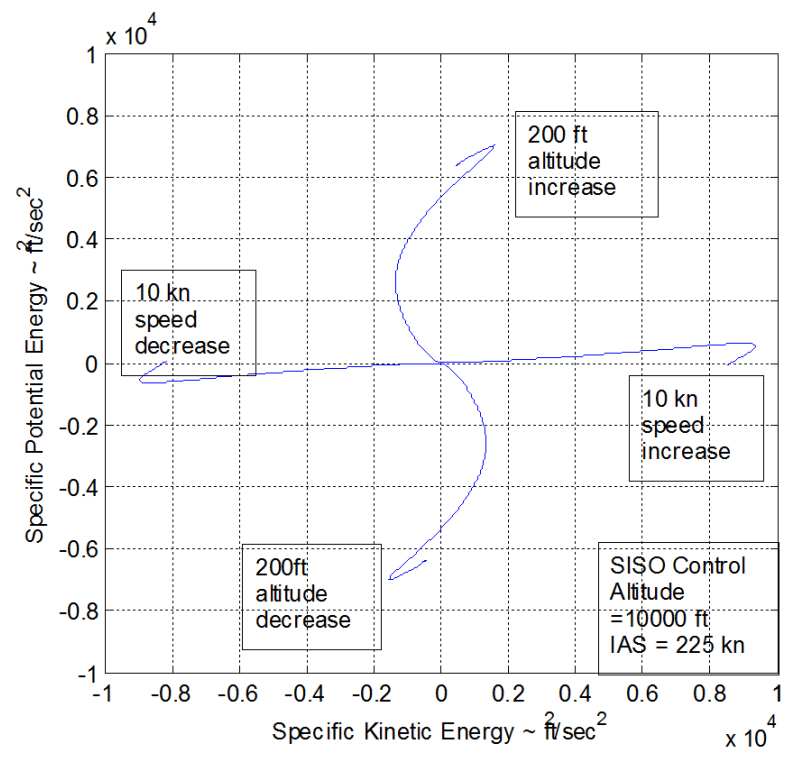

Figure 2. Energy Path to New State for a SISO Type Control System

Separate numerical simulations were used to obtain the energy state transition response to a step altitude command input and to a step airspeed command input. Clearly, the transitions do not take place along a direct potential or kinetic energy paths, but take a control coupling route involving undesirable temporary energy transfer.

In [6], the misconception underlying conventional flight path on elevator and speed on throttle SISO control strategy is described in the following way: 'many persons not dealing professionally with stability and control fall back on the common usage of the terms elevator and throttle and believe the elevator mainly raises or lowers the aircraft and the throttle speeds or slows it. Really it is the other way around. The elevator is the primary speed control of the airplane and the throttle setting determines whether the airplane rises or descends at a given speed'.

The limitations of specific SISO modes often became the driving force for the design of yet more specialized modes and sub-modes. Thus, the complexities of the current systems are, to a large extent, attributable to the inherent shortcomings of single input/single output (SISO) control strategies. The design and operational complexities increased even more when the first all-axes automatic Flight guidance and Control System (FGCS) and Flight Management Systems (FMS) were introduced, ...with more unsatisfactory results [7].

\section{TECS MIMO Control}

In the mid- to late seventies, NASA conducted extensive Terminal Area Guidance and Control research under the Terminal Configured Vehicle (TCV) program that included among other subjects the application of digital flight control computers, MLS guidance, advanced control laws (autoland, LNAV, VNAV, 4D, etc.), early flight management computers, and electronic displays. Based on this work it was recognized that in order to fully address the SISO control related problems, an advanced MIMO based FGCS would be needed. In this FGCS all modes of operation are functionally integrated, based on a common flight guidance and control design strategy and operations concept. This prompted NASA to sponsor research at Boeing to develop a generalized, functionally integrated Multi-Input/Multi-Output (MIMO) guidance and control system architecture for the vertical flight path and airspeed control modes [8]. This work resulted in Total Energy Control System (TECS). Likewise, the research and development of a generalized functionally integrated Multi- 
Input/Multi-Output (MIMO) control system architecture for the lateral-directional control modes was conducted at Boeing under DARPA sponsorship during the Condor High Altitude Long Endurance (HALE) Technology Demonstration program. TECS was first flight tested in 1985 on the NASA B737 airplane. Both TECS and THCS were very successfully applied and flight tested on the Condor airplane (1984-1990) [9].

TECS $[8,10]$ provides a full array of functionally integrated automatic and augmented manual control modes for airplane control in the vertical plane. Likewise, THCS [7] provides all needed functions for automatic and augmented manual control of the airplane in the horizontal plane. Figure 3 shows the TECS core concept.

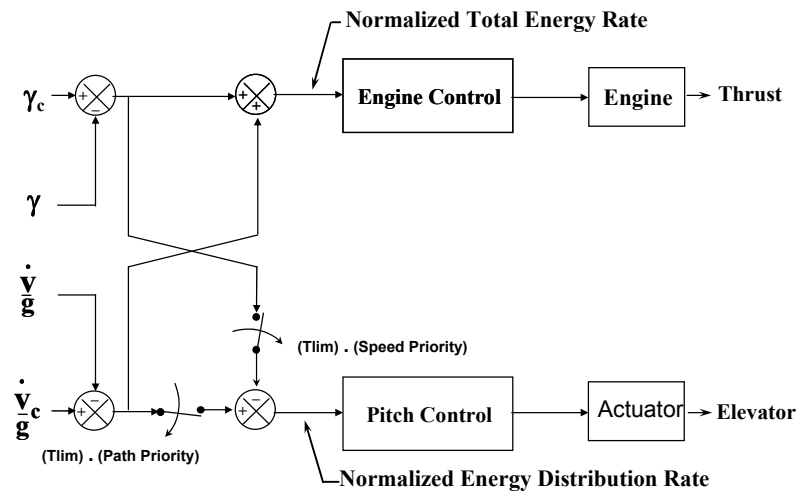

Figure 3. Total Energy Core Control Concept

The key difference between the SISO control architecture of Figure 1 and the MIMO architecture of the TECS core control concept of Figure 3 is that the control errors $\left(\gamma_{c}-\gamma\right)$ and $\left(\dot{\mathrm{v}}_{\mathrm{c}} / \mathrm{g}-\dot{\mathrm{v}} / \mathrm{g}\right)$ are cross fed to each controller. Thrust controls the sum of the error signals (energy-rate), while the elevator controls the difference between the error signals (energy redistribution rate). As a condition for achieving decoupled flight path command and speed command responses the response dynamics of $\left(\gamma_{\text {error }}+\dot{\mathrm{v}}_{\text {error }} / \mathrm{g}\right)$ must be equal to the response dynamics of $\left(\gamma_{\text {error }}-\dot{\mathrm{v}}_{\text {error }} / \mathrm{g}\right)$. For any outer-loop mode (not shown in Figure 3 ) the actual path control error is normalized into a $\gamma_{\mathrm{c}}$ command signal and the speed control error is normalized into $\mathrm{a} \dot{\mathrm{v}}_{\mathrm{c}} / \mathrm{g}$ command signal, serving as inputs to the TECS core controller. To prevent the airplane from departing the safe speed envelope, the switches shown in Figure 3 are used to establish elevator control priority when thrust reaches a limit. In addition, simple provisions are implemented (not shown) to limit the load factor and to assure energy efficient execution of any combination of simultaneous flight path and speed commands, even when thrust is at a limit. The generalized TECS architecture results in an overall simpler, reusable design that enhances automation safety. It provides improved and consistent performance for all mode combinations. Due to this consistency (fewer special modes), it also has allowed the pilotmachine interface design to be simplified.

Figure 4 shows the simulation result of the energy response of the TECS MIMO control system to an altitude command and to a speed command. Clearly, these responses are much better decoupled than the responses for a typical SISO system shown in Figure 3. In Figure 4, the small change in kinetic energy at the end of the altitude change results from the increase in true airspeed with a change in altitude at constant indicated airspeed.

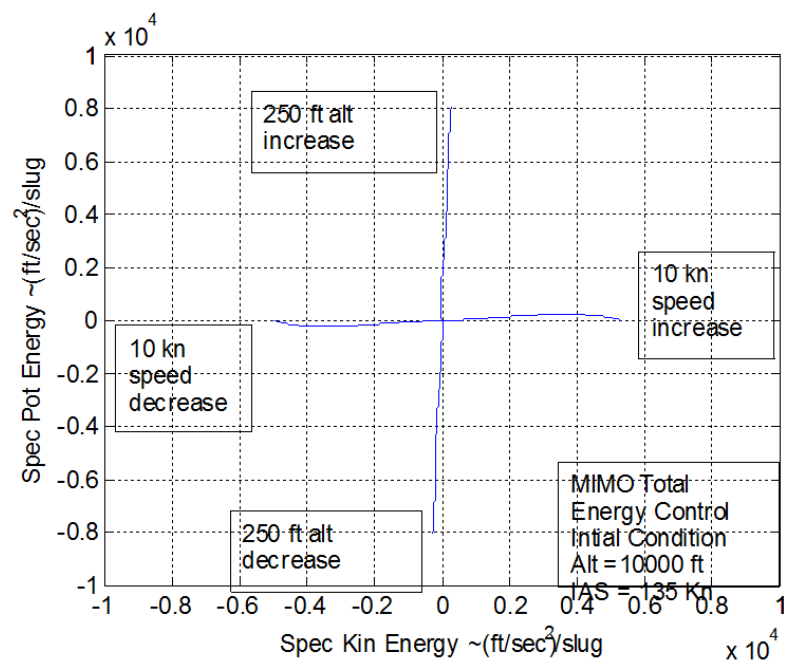

Figure 4. Energy Path to a New State for MIMO System

Unfortunately, generalized MIMO based FCGS designs, like TECS/THCS, have still not been used on production airplanes, possibly due to industry inertia in making a large paradigm shift and the perceived application risk. 


\section{Airplane Control from an Energy Perspective}

To aid in the understanding of the subsequent description of the Energy Management PFD concept, a derivation of the airplane energy relationships and a discussion of energy based control strategy is presented.

Consider the equations (1) and (2) for airplane dynamics along the flight path and perpendicular to the flight path:

$$
\begin{aligned}
& \text { (1) } \frac{\mathrm{W}}{\mathrm{g}} \dot{\mathrm{V}}=\mathrm{T}-\mathrm{D}-\mathrm{W} \sin \gamma \cong \mathrm{T}-\mathrm{W}\left(\frac{\mathrm{C}_{\mathrm{d}}}{\mathrm{C}_{1}}-\gamma\right) \text {, or } \\
& \text { (1a) } \frac{\mathrm{T}-\mathrm{D}}{\mathrm{W}}=\frac{\dot{\mathrm{v}}}{\mathrm{g}}+\gamma=\gamma_{\mathrm{P}} \\
& \text { (2) } \frac{\mathrm{W}}{\mathrm{g}} \mathrm{V} \dot{\gamma}=\mathrm{L}-\mathrm{W} \cos \gamma
\end{aligned}
$$

Where:

$$
\begin{aligned}
& \text { W }=\text { airplane weight } \\
& \mathrm{T}=\text { airplane thrust } \\
& \mathrm{D}=\text { airplane drag, } \mathrm{C}_{\mathrm{d}}=\text { drag coefficient } \\
& \mathrm{L}=\text { airplane lift, } \mathrm{C}_{1}=\text { lift coefficient } \\
& \mathrm{V}=\text { airplane velocity } \\
& \dot{\mathrm{V}}=\text { airplane longitudinal acceleration } \\
& \text { along } \quad \text { the flight path, relative to inertial space } \\
& \gamma=\text { FPA, relative to air mass } \\
& \gamma_{\mathrm{P}}=\text { PFPA } \\
& \dot{\gamma}=\text { rate of change of FPA } \\
& \mathrm{g}=\text { gravity constant }
\end{aligned}
$$

In the equilibrium condition $\dot{\mathrm{v}} / \mathrm{g}=0$ and thrust is trimmed against $\mathrm{D}$ and $\gamma$. Use of the elevator causes the airplane to rotate about its pitch axis, resulting in a change in angle of attack and lift. By definition, lift is perpendicular to the flight path and therefore does not directly affect the airplane's total energy. Because airplanes are preferably flown at an angle of attack where $\mathrm{C}_{\mathrm{d}} / \mathrm{C}_{1}$ is close to the minimum, for gentle pitch maneuvers at constant thrust, the change in $\mathrm{W} \cdot \frac{\mathrm{C}_{\mathrm{d}}}{\mathrm{C}_{1}}$ is negligibly small compared to $\mathrm{W} \cdot \Delta \gamma$. For such elevator-only maneuvers it follows that

(1b) $\frac{\mathrm{T}-\mathrm{D}}{\mathrm{W}}=\frac{\dot{\mathrm{v}}}{\mathrm{g}}+\gamma \cong$ Constant .

Therefore $\frac{\dot{\mathrm{v}}}{\mathrm{g}} \cong-\gamma$

Thus, application of the elevator at constant thrust causes a change in the longitudinal acceleration $\dot{\mathrm{v}} / \mathrm{g}$ which is equal and opposite to the change in FPA $(\gamma)$. To change the magnitude of $\left(\frac{\dot{\mathrm{V}}}{\mathrm{g}}+\gamma\right)$ requires in essence a thrust change. The quantity $\left(\frac{\dot{\mathrm{v}}}{\mathrm{g}}+\gamma\right)$ is the PFPA $\left(\gamma_{\mathrm{p}}\right)$, which is the flight path angle the airplane attains when the rate of change of the velocity is reduced to zero by applying elevator/pitch control. For this reason $\gamma_{p}$ has been used in Head-Up Displays (HUDs), together with $\gamma$, to provide an effective thrust guidance cue.

Considering the Specific Total Energy $\mathrm{E}_{\mathrm{s}}$ :

(3) $E_{s}=\frac{V^{2}}{2}+g h$, where $h$ is the altitude, it follows that

(4) $\frac{\dot{\mathrm{E}}_{\mathrm{s}}}{\mathrm{gV}}=\frac{\dot{\mathrm{v}}}{\mathrm{g}}+\gamma=\gamma_{\mathrm{p}}$

Thus, $\left(\frac{\dot{\mathrm{v}}}{\mathrm{g}}+\gamma\right)$ is a measure of the

(dimensionless) Total Energy rate. Thrust is therefore most effectively used as an energy controller, rather than an airspeed controller.

Since the elevator in effect only causes a trade between $\dot{\mathrm{v}} / \mathrm{g}$ and $\gamma$, it redistributes the balance between kinetic and potential energy, or velocity and altitude. The elevator is therefore most effectively used as an energy redistribution controller. A measure of the rate of energy redistribution may be defined as $\dot{\mathrm{F}}$, where 
(5) $\dot{\mathrm{F}}=\frac{\dot{\mathrm{V}}}{\mathrm{g}}-\gamma$

In classical mechanics $\dot{\mathrm{F}}$ is referred to as the LaGrangean. Using $\frac{\dot{\mathrm{E}}_{\mathrm{s}}}{\mathrm{gV}}$ for thrust control and $\dot{\mathrm{F}}$ for pitch control is an ideal generalized MIMO control strategy, because when the control dynamics of $\frac{\dot{\mathrm{E}}_{\mathrm{s}}}{\mathrm{gV}}$ and $\dot{\mathrm{F}}$ are designed to be identical, it results in perfectly decoupled flight path and speed command control responses with a "minimum control effort", or minimum wasted energy. For this reason this strategy was used in the development of the TECS integrated vertical flight path and airspeed automatic guidance and control system.

\section{Designing a PFD from an Energy Perspective}

Regarding the concept of conservation of energy, Feynman [11] states 'The conservation of a physical property is of considerable interest because in solving problems it permits us to forget a great number of details. The conservation of energy can be derived from the laws of motion, but its value lies in the fact that by the use of it certain broad aspects of a problem may be discussed without going into great detail that is often required by a direct use of the laws of motion'.

In this section, it will be discussed how energy awareness can contribute to the selection of control actions without requiring the pilot to rely on an accurate internal model of the system under control. Figure 5 presents an energy graph for two state transitions (new altitude and new speed). The scaling in terms of energy on the $\mathrm{x}$ - and $\mathrm{y}$-axis is the same, hence lines at an angle of -45 degrees all represent states that have an equal amount of total energy, i.e. the sum of $E_{k}$ and $E_{p}$ is constant along such a line. In Figure 5, the transition to a new altitude while maintaining constant speed can be plotted as a vertical line from the current state 1 to a new state 2 . The transition to a new speed while maintaining altitude can be plotted as a horizontal line from state 1 to state 3 . From the way the throttle and elevator influence the energy it can be seen that the transition from one speed to another (while maintaining altitude) requires the use of both thrust and elevator. The same holds for a transition from one altitude to another (while maintaining speed). In the ideal case, the control system commands the elevator deflection and the engine thrust in such a way that the sum of the energy vectors lies along a straight line to the new target state. The elevator changes the location of the point along a line of constant energy.

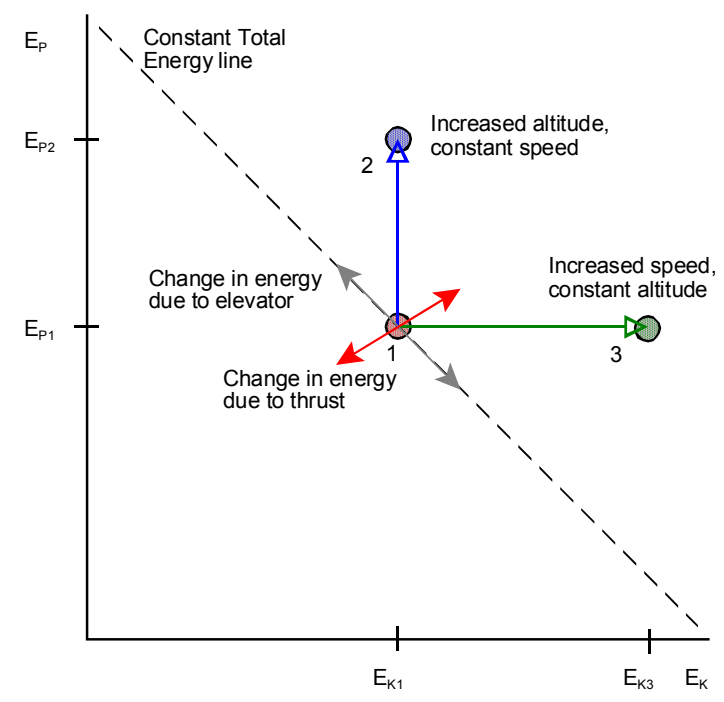

Figure 5. Energy Graph

The amount of energy added/subtracted to/from the system is largely determined by the engine. In the previous subsections discussing SISO control and MIMO control, it has been shown how the energy trajectory for the conventional SISO control strategy (Figure 2) deviates far more from the desired straight line than for the TECS Energy control strategy (Figure 4).

\section{Providing the Pilot with Energy Awareness}

As illustrated, based on energy principles, a control scheme can be devised that allows an optimal transition between different speed and altitude targets. This raises the question whether it is possible to provide the pilot with information on the PFD that supports such a control strategy. The instruments on a typical conventional PFD (Figure 6) provide information on the different SISO signals in Figure 1. In terms of Situation Awareness (SA), the information that is provided can be classified as level 1 (perception of the separate elements). Integration (needed to achieve level 2) and extrapolation, needed to exercise 
control based on the desired future state (level 3) has to be performed by the pilot.

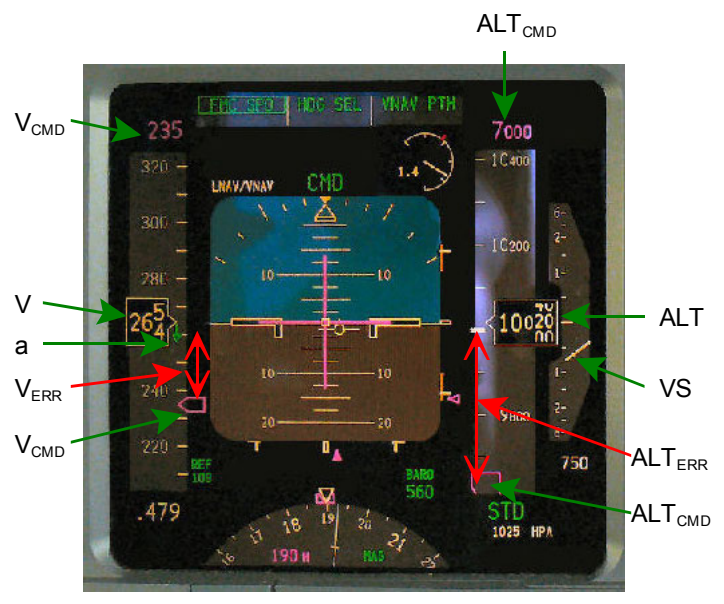

\section{Figure 6. Example of a PFD Format}

With the introduction of the PFD, the main goal was to replace a number of electromechanical instruments with a single display. This, by itself, posed the designer with considerable challenges. Konicke [1] describes five different formats that were evaluated as candidates for the 747-400 PFD. Design questions focused on the representation (round dial fixed scale versus vertical tape moving scale indicators), the effect of readouts obscuring the scales, and the locations of the different instruments. Regarding the altitude tape and the vertical speed indicator, the following remark is made: 'it offered a degree of integration in the area of altitude and vertical speed that was not present with any other PFD format. Although the altitude and vertical speed instruments were still separate, the proximity of the instruments to one another enabled the pilots to perceive easily how vertical speed information relates to desired altitude targets in the vertical plane. The moving pointer vertical speed indicator will move up or down to show a trend towards or away from the selected altitude cursor or tape numerics, giving the pilot the ability to determine the goodness of level-offs and other altitude trends'.

A similar comment is made about the trend vector which displays where airspeed will be in 10 seconds and it is concluded that 'the benefit of integrating altitude and vertical speed data while maintaining the basic T scan pattern appeared to be worth the possibility of additional flight training (needed to go from round dial to tape instrument)'. This illustrates how appropriate location and scaling of different instruments can be used to support the pilot in obtaining level 2 and level 3 SA. To illustrate how energy awareness is needed to select the right control actions, Figure 7 shows five example situations for a new potential and kinetic energy state.

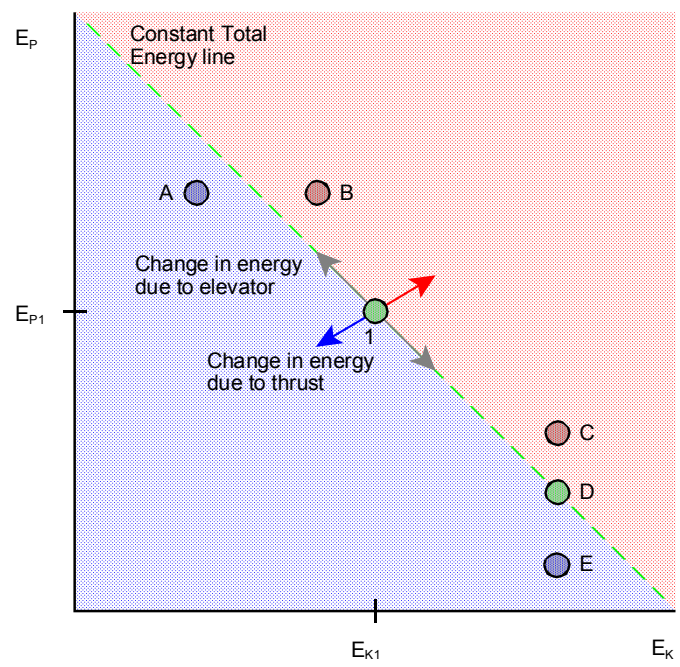

Figure 7. Energy States of Targets A to E

Both states A and B represent situations of increased target altitude and reduced target speed relative to the current state (1). The difference between states A and B is that in terms of total energy, state A has a lower total energy than the current state and state B has a higher total energy. Hence, to transition to state $\mathrm{A}$, the pilot will have to pitch up and reduce thrust, whereas a transition to state $\mathrm{B}$ requires a pitch up and an increase in thrust. Points C, D and E all represent target states with a higher speed and a lower altitude. Target state D lies on the line of constant total energy that also runs through the current state. This indicates that target state $\mathrm{D}$ can be reached by exchanging potential energy for kinetic energy using elevator control only. No thrust control is needed in this case. Target state $\mathrm{C}$ represents a situation where thrust needs to be increased and target state $\mathrm{E}$ represents a situation where thrust needs to be reduced.

To support a control strategy based on energy awareness, a display would need to provide explicit information about energy differences to the pilot that can be used to distinguish between situations $\mathrm{A}$ and $\mathrm{B}$, and between C, D, and E in Figure 7. 
Furthermore, the display would need to provide guidance on how to use the controls to influence the total energy and energy distribution to reach the desired state. Using the energy relations as shown in Figure 7, the SA can go from level 1 to level 2. By also providing information about the future energy state, level 3 can be reached. In [12], a design approach that is based on an analysis of the different SA levels is referred to as 'design for situation awareness'. The goal of the enhanced PFD is to support the pilot in obtaining level 3 awareness concerning flight path and speed. The name used for such a display in the remainder of this paper is Energy Management PFD (EMPFD).

\section{The Energy Management PFD Concept}

\section{Visualizing Energy Error and Rate}

From the equations in the previous section, it follows that to directly convey the control input requirement to move to desired flight path and speed target states, the kinetic and potential energy errors, the kinetic and potential energy rates, the total energy error and the rate of energy distribution information need to be visualized in the display. Obviously, this can be achieved through the addition of new instruments to represent potential and kinetic energy errors. However, rather than adding more instruments, the required energy error and rate information can be conveyed through appropriate scaling and formatting of the current display variables (speed, speed command, rate of change of speed, altitude, altitude command and rate of change of altitude).

Figure 8 presents a conceptual energy scaled display for the depiction of speed, acceleration, vertical speed and altitude. The left green bar represents the speed error and the right green bar the altitude error. The blue bars represent rate of change of speed and vertical speed. The scaling of the speed tape is defined in kinetic energy/inch and the scaling of the altitude tape in potential energy/inch. By using equal scaling in terms of energy/inch, comparison of speed- and altitude errors in terms of energy becomes possible at a glance. Likewise, to be able to extract control guidance information, both rate scales need to be normalized to the same energy-rate/inch.

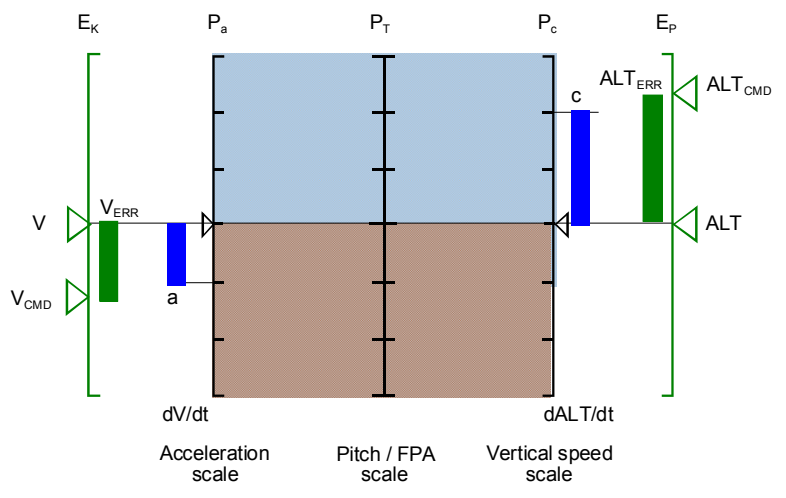

Figure 8. Conceptual Energy Scaled Display

For the situation depicted in Figure 8, from the difference in length between the bars it can be concluded that the surplus in kinetic energy is not sufficient to compensate for the shortage in potential energy. Hence, to reach the desired altitude, the pilot will have to add power. The blue bar on the left represents the rate at which the airplane loses kinetic energy through deceleration. The blue bar on the right represents the rate at which the airplane gains potential energy by climbing.

Note that compared to the layout of a conventional PFD, the location of the vertical speed indicator has moved from the right of the altitude tape to the left. Regarding deviations from the basic $\mathrm{T}$ that have been substantiated by satisfactory service experience and research [3] mentions the 'vertical scale display of vertical speed between attitude and altitude displays' is also acceptable.

The ratio between the energy scales and the energy-rate scales is a time constant. If the controls are used such, that the rate indications line up with $\mathrm{V}_{\mathrm{CMD}}$ and $\mathrm{ALT}_{\mathrm{CMD}}$, this time constant represents the first order approximation of the energy response capturing $\mathrm{V}_{\mathrm{CMD}}$ and $\mathrm{ALT}_{\mathrm{CMD}}$ targets. Thus, by selecting an appropriate value for the speed and altitude capture guidance time constant (e.g. 10 seconds), the display provides control cuing information for capturing the desired altitude and speed targets.

\section{Visualizing the Distribution of the Energy- Rate}

An awareness of the energy errors and their rate of change is the first prerequisite for efficient control. The second prerequisite is information on 
how much additional energy is being added and how it is distributed. These energy awareness cues enable the pilot to distinguish the different relative elevator- and thrust control actions needed to reach the targets A and B and between C, D and E in Figure 7.

Use of the elevator control will only cause an exchange of energy between speed and altitude and cause the acceleration and vertical speed indications to move in opposite directions. Therefore, either the acceleration can be changed to line up with the speed command, or the vertical speed can be lined up with the altitude command to capture one of these targets by the use of the elevator. The needed energy will be taken from the other variable, unless thrust is changed appropriately. Alternatively, the elevator can be used to equalize the magnitudes of the acceleration and the vertical speed errors relative to their associated command targets. Then, by applying thrust, both the acceleration and the vertical speed indication bars will move the same amount in the same direction, thereby allowing both bars to be lined up with their associated commands. Thus, in principle the energy and energy-rate indications in Figure 8 provide both elevator- and thrust control cueing information to simultaneously capture speed and altitude targets with the guidance time constant built into the display.

The addition of the FPA $(\gamma)$ and PFPA $\left(\gamma_{P}\right)$ display symbols in the center, using the same scale as pitch attitude (as is now customary in most HUDs), will further enhance the pilot's capability to manage the flight path and set thrust as needed to capture flight path and speed targets. As shown in Eq. 4, the PFPA $\left(\gamma_{P}\right)$, is indicative of the total amount of energy being added (with thrust). The way this energy is being distributed, as controlled by the elevator, is indicated by the FPA $(\gamma)$, vertical speed, and the acceleration. This relation can be visualized through a depiction of $\gamma_{P}$ and $\gamma$ relative to the acceleration and the vertical speed scale. The following three example situations visualize this relation. In these examples, $\gamma_{\mathrm{P}}$ is indicated by the PFPA marker and $\gamma$ by the FPA marker.

Figure 9 illustrates the situation where the airplane is in a climb at constant speed, i.e. the aircraft uses all excess energy-rate to climb. Since acceleration $(\dot{\mathrm{V}} / \mathrm{g})$ is zero, $\gamma$ is equal to $\gamma_{\mathrm{P}}$, hence the PFPA marker is at the same location as the FPA marker. If the appropriate scaling is used, the location of the PFPA marker (indicated by the red circle) and the FPA marker (indicated by the blue dot) on the pitch scale lie on a straight line between the current acceleration and the current vertical speed.

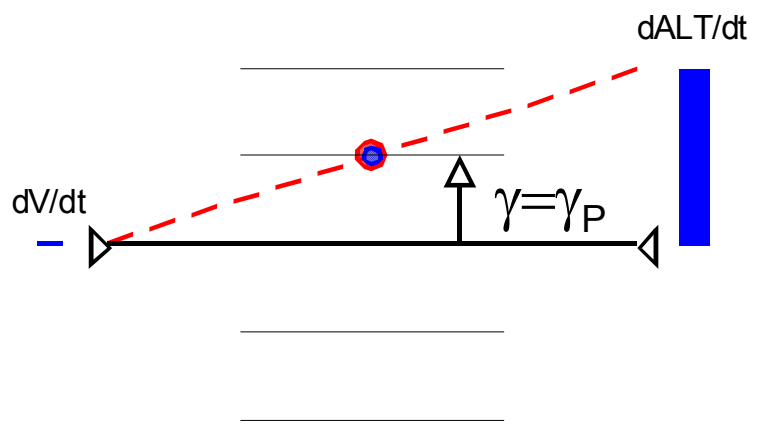

Figure 9. All Excess Energy is Used to Climb and $\gamma_{\mathrm{P}}$ is Equal to $\gamma$

This is illustrated by the dashed line drawn between the acceleration indication and the vertical speed indication. It shows how energy is being distributed.

Figure 10 shows a situation with the same thrust as in Figure 9, hence the PFPA marker is still in the same location.

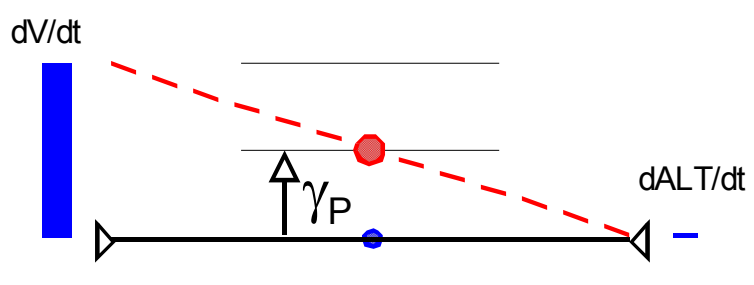

\section{Figure 10. All Excess Energy is Used to Accelerate}

The total amount of energy added to the system has remained the same but the elevator has been used to alter the distribution of the energy and convert it solely into acceleration. The normalized acceleration $(\dot{\mathrm{V}} / \mathrm{g})$ is now equal to the PFPA $\left(\gamma_{\mathrm{P}}\right)$ (i.e. all of the excess energy-rate is used to accelerate) and the aircraft is in level flight, indicated by the location of the FPA marker (blue circle) on the horizon line. Note that the PFPA marker now lies on a line between the current 
vertical speed indication (zero) and the current acceleration.

Considering both Figure 9 and 10, it can be observed that the red PFPA marker remained in the same vertical location, since there was no change in thrust and that the PFPA marker also serves as the pivot-point of the dashed line. The rotation of the dashed line is controlled by the elevator and its tilt angle indicates how the energy-rate is being distributed.

Figure 11 illustrates a situation where the thrust is trimmed for level flight, hence the PFPA marker is on the horizon line. The dashed red line shows that kinetic energy is being converted into potential energy (redistribution). The actual FPA marker is on the center of the black dashed line, connecting the zero acceleration marker and the current vertical speed.

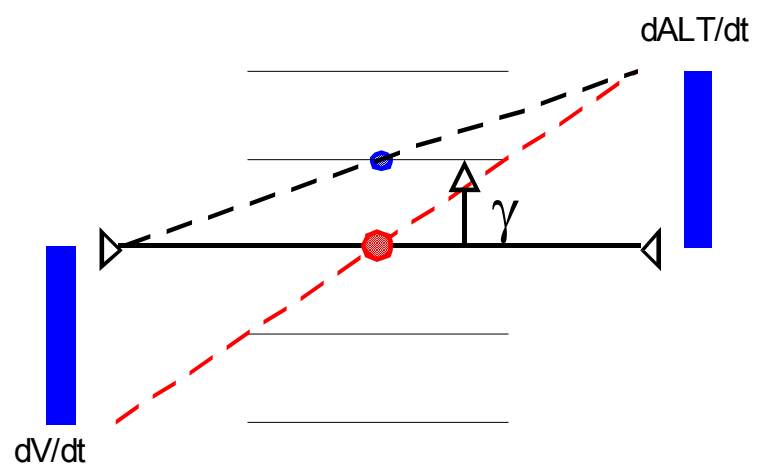

Figure 11. Conversion of $E_{k}$ into $E_{p}$

When integrating this visualization concept with the display shown in Figure 8, the display shown in Figure 12 results.

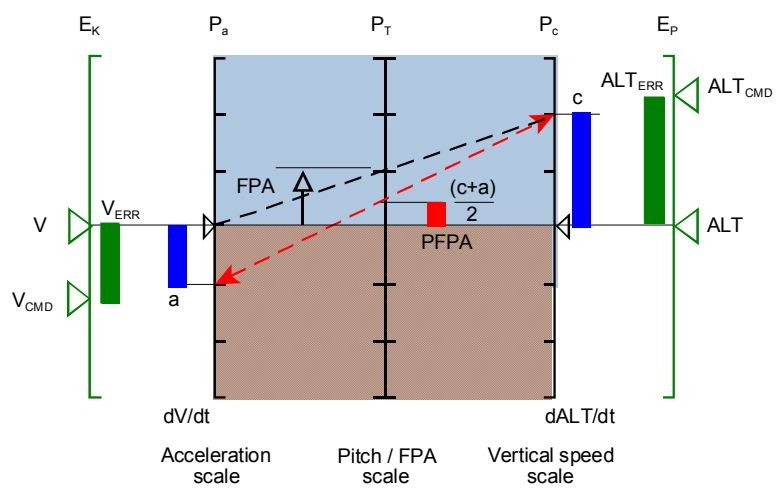

Figure 12. Conceptual EMPFD Format

The vertical distance (indicated by the red bar) between the intersection of the red dashed line with the pitch scale and the horizontal reference line is proportional to the excess thrust beyond the thrust level needed to maintain level flight at constant speed.

\section{Providing Guidance Information}

As mentioned earlier in this paper, Konicke [1] already reports on advantages resulting from the spatial proximity of the vertical speed indicator and the altitude tape to provide "trend" information. The speed "trend" vector in today's electronic PFDs fulfills a similar function. In the EMPFD here discussed, the blue energy-rate bars also provide speed- and altitude "trend" information. However, the energy-rate bars are placed on the inside of the speed- and altitude energy error bars and all four bars are scaled consistently in terms of energy-rate and energy error per unit length to provide the pilot with directly actionable control guidance information. If the ratio of the energy/inch and energy-rate/inch scales is selected at 10 seconds, the length of the blue acceleration bar represents the change in speed or altitude that will be obtained in 10 seconds. For example, if the speed scale indicates a $10 \mathrm{kts}$ difference between $\mathrm{V}_{\mathrm{CMD}}$ and $\mathrm{V}$, then when the blue acceleration bar is lined up with $\mathrm{V}_{\mathrm{CMD}}$ the acceleration indication is $1 \mathrm{kts} / \mathrm{sec}$. Likewise, if the altitude scale indicates a $100 \mathrm{ft}$ difference between ALT $_{\mathrm{CMD}}$ and ALT, then when the blue vertical speed bar is lined up with $\mathrm{H}_{\mathrm{CMD}}$ the vertical speed indication is $10 \mathrm{ft} / \mathrm{sec}$, or $600 \mathrm{ft} / \mathrm{min}$.

\section{Capturing Speed and Altitude Targets}

As explained earlier, if the pilot keeps the blue bars lined up with the command indications on the green bars, the commands will be captured with dynamics that approach a first order lag response with a time constant equal to the ratio of the energy /inch and energy-rate/inch scales. This ratio therefore represents the command capture guidance time constant.

In order to facilitate capturing $\mathrm{V}_{\mathrm{CMD}}$ and $\mathrm{ALT}_{\mathrm{CMD}}$ simultaneously, another guidance symbol is needed to indicate where to put the PFPA marker using thrust adjustments during the capture. This Thrust Setting Marker (TSM) must be displayed in the center of the line that connects points, on left and right extremities of the center pitch indication square, that line up horizontally with the vertical position of the $\mathrm{V}_{\mathrm{CMD}}$ and the ALT $\mathrm{CMD}_{\mathrm{C}}$. This extra 
guidance symbol is not needed to capture an $\mathrm{H}_{\mathrm{CMD}}$ while holding the speed constant. In that situation, the pilot uses the elevator during the altitude capture to keep the vertical speed lined up with $\mathrm{H}_{\mathrm{CMD}}$ and adjusts the thrust to keep the PFPA marker lined up with the resulting FPA.

\section{Tracking Path and Speed}

On a PFD, guidance information for precision control (i.e. tracking a predefined path) is typically provided by means of a flight director. The flight director command is based on a weighted sum of position and/or angular errors and their rates. The design of optimal flight director algorithms focuses on identifying the appropriate gains [13] to drive the command bar. Philosophically, the FD automation approach, starting from the outer loops and developing a high-frequency command bar that the pilot must follow, is backward, because it creates high pilot workload and leaves the pilot less aware of the airplane flight dynamics information provided on the basic PFD. In contrast, the EMPFD helps the pilot to stay focused on all important airplane dynamics variables and their relationships. In addition, it provides guidance cues for vertical flight path and speed control, using symbols that have a physically interpretable meaning and are already in use on many PFDs. Ideally, automation should start with the inner loop to provide pilot relief of high frequency workload, allowing the pilot to manage the outer loops that control where the airplane is going. The EMPFD adheres to this principle, by allowing the pilot to bypass pitch attitude control, and use, to a certain extent, an inner-loop error-neglecting strategy. In other words, rather than use a continuous target tracking strategy, the pilot can adopt an intermittent control strategy, and still zero-in on the outer-loop speedand altitude targets without overshooting. It should be noted that direct manual FPA control without using a pitch attitude as an intermediate control variable can lead to poor handling qualities and PIO but this can be avoided by using FPA-display quickening $[14,15,16]$.

A very important benefit of the FPA indication is that it represents the airplane's velocity vector and therefore it predicts the airplane's future position relative to surrounding objects in inertial space. On a HUD and a Synthetic Vision Display, the visualization of terrain features provides the pilot with timely awareness of flight paths that conflict with terrain. During approach and landing, the FPA display overlayed on the real or synthetic approach terrain provides the pilot with glide path and landing guidance cues. In this regard, Newman [17] states: 'The Klopfstein synthetic runway has been reported to produce excellent tracking performance even without the use of a flight director and, at the same time, to permit a high degree of spatial orientation'.

Unlike the typical PFD, HUDs do present FPA and most often also an FPA target cue. This pilot settable target cue is usually represented by a dotted horizontal line, It is also referred to as a flight path marker (FPM). In fact, Newman [17] reports that 'All known implementations of such steering cues have been referenced to the FCDM/FPM (flight path command marker/ flight path marker). This (use of FCDM/FPM) has been proposed as an Air Force standard'.

On most HUDs, the FPA marker is accompanied by so a called acceleration cue (often represented by means of a caret) which indicates the airplane's acceleration and its magnitude is projected relative to the FPA marker, using the same energy-rate/inch scaling as used for the FPA marker. Therefore, this cue also represents an indication of the PFPA and has the same function as the PFPA marker described here for the EMPFD.

\section{EMPFD Feasibility}

Until now, only a hypothetical display has been presented. It may very well be that the requirement of equal scaling in terms of energy may result in impractical ranges or resolutions for one or more of the scales used in a EMPFD. Hence, the first question that needs to be answered is whether such a display format is feasible in terms of scaling. To address this question, the equations that relate the scales have to be derived. From these equations it can be determined whether sufficient degrees of freedom exist to realize the desired scaling. If so, the next question is whether the scaling is practical, i.e. can values for the scaling of all instruments be selected that provide the required range and adequate sensitivity. The final question is whether the display is suitable and effective and this must be answered by pilot-in-the-loop evaluation and task performance analyses, using an 
appropriate set of tasks and suitable performance metrics.

\section{Deriving the Scaling Equations}

In the previous sections we discussed expressing speed and altitude with an energy/inch scaling factor. It is noted here that, because the pitch attitude and FPA scale is linear, the acceleration scale in Figures 10, 11 and 12 must be linear in terms of knots (true airspeed/second) /inch. Since the ratio between the scales of speed and acceleration must be a constant (the speed control guidance time constant), the underlying speed scale will also need to be linear in terms of (knots true airspeed )/inch, rather than linear in terms of energy/inch. The ramification of choosing a background speed scale that is linear in terms of $\mathrm{V}_{\text {TRUE }} /$ inch is that the altitude and vertical speed scales must use scalings that are compatible, and this will be discussed below. The actual speed and acceleration scales will indicate Calibrated Airspeed and Calibrated Airspeed/second and these scales will be slightly non-linear, and compressing (more $\Delta \mathrm{V}_{\mathrm{CAS}} /$ inch and $\Delta \mathrm{V}_{\mathrm{CAS}} / \mathrm{sec} /$ inch) with increasing altitude. The $\mathrm{V}_{\mathrm{CAS}}$ scale is computed by converting the $\mathrm{V}_{\text {CAS }}$ scale tick marks into $\mathrm{V}_{\text {TRUE }}$ values for a given flight condition, and expressing these $V_{\text {TRUE }}$ values on the linear $V_{\text {TRUE }}$ scale. The scale compression of $\mathrm{V}_{\mathrm{CAS}} / \mathrm{V}_{\mathrm{TAS}}$ is 1.0 at sea level and $\sim 1.70$ at $\mathrm{V}_{\mathrm{CAS}}=250 \mathrm{kts}$ and $35000 \mathrm{ft}$ altitude.

The display requires three basic scaling factors: the acceleration scaling factor $\left(S_{a}\right)$, the pitch / FPA scaling factor $\left(\mathrm{S}_{\gamma}\right)$ and the vertical speed scaling factor $\left(\mathrm{S}_{\mathrm{c}}\right)$. Since FPA, PFPA $\left(\gamma_{\mathrm{P}}\right)$ and pitch attitude $\theta$ share the same scale, $S_{\gamma}=S_{\gamma_{\mathrm{P}}}=S_{\theta}$. The scales for the remaining two variables (speed and altitude) follow from acceleration and vertical speed scaling factors and the guidance time constant $\tau$ defined by the ratio of the energy and energy-rate scales.

If $a$ represents the length of the acceleration indication bar, $c$ represents the length of the vertical speed indication bar, then

$$
a=S_{a} \cdot \frac{d V}{d t},(c+a) \frac{1}{2}=S_{\gamma_{P}} \cdot \gamma_{P}, c=S_{c} \cdot \frac{d h}{d t}
$$

Substitution yields:
(6) $S_{a} \cdot \frac{d V}{d t}-2 S_{\gamma_{P}} \cdot \gamma_{P}+S_{c} \cdot \frac{d h}{d t}=0$

FPA can be expressed as: $\gamma=\frac{1}{V} \frac{d h}{d t}$

Considering $\gamma_{\mathrm{P}}=\gamma+\dot{\mathrm{v}} / \mathrm{g}$, by setting $\dot{\mathrm{V}} / \mathrm{g}=0$, so $\gamma_{\mathrm{P}}=\gamma$, the relationship between the scaling factor of the vertical speed scale and the scaling factor of the FPA scale can be determined by substituting $\dot{\mathrm{V}} / \mathrm{g}=0$ and $\gamma_{\mathrm{P}}=\gamma$ into (6):

$$
S_{c}=\frac{2}{V} S_{\gamma}
$$

Likewise, the relationship between the scaling factor of the acceleration scale and the scaling factor of the FPA scale can be determined by substituting $\dot{\mathrm{v}} / \mathrm{g}=\gamma_{\mathrm{P}}$ and $d h / d t=0$ into (6) yielding:

$$
S_{a}=\frac{2}{g} S_{\gamma}
$$

From these equations, it can be observed that the relation between the FPA scale and the acceleration scale is constant.

However, the ratio between the FPA scale factor and the vertical speed scale factor, and consequently also the ratio between the vertical speed and the acceleration scale factors is proportional to velocity. It also implies that, in order to maintain the desired fixed scaling ratio between the altitude and vertical speed scales, the altitude scale must be made proportional to the velocity.

Equal $\Delta$ kinetic and $\Delta$ potential energy must be represented by equal bar lengths on the speed and altitude scales. The kinetic energy represented by a given $\Delta \mathrm{V}_{\text {TRUE }}$ can be converted into the equivalent potential energy using the Total Energy equation $(1 / 2) \times V^{2}+g \times h=$ constant. Starting from an initial condition with $\mathrm{V}_{0}$ and $\mathrm{h}_{0}$, one finds

(7) $\Delta h=-\frac{V_{0} \cdot \Delta V+(\Delta V)^{2}}{g}$ 
Using this non-linear relationship would be in conflict with the above scaling requirements. Linearization of equation (7) results in

(8) $\Delta \mathrm{h}=-\frac{\mathrm{V}_{0}}{\mathrm{~g}} \Delta \mathrm{V}$

Equation (8) meets the above relative altitude and speed scaling requirements and therefore this linearized equation (8) must be used, even though it does not represent an exact energy conversion. The largest mismatch of relative energy between the speed and altitude scales will occur for full range altitude indication at the lowest $\mathrm{V}_{\text {TAS }}$. This energy mismatch is equivalent to a $\sim 25 \%$ too high a scale factor (inch/ $\Delta$ altitude) for the altitude indication scale at $\mathrm{V}_{\text {TAS }}=120 \mathrm{kts}$ and $6.7 \%$ too high a scale factor at $\mathrm{V}_{\mathrm{TAS}}=550 \mathrm{kts}$. This is not expected to be a significant problem in practice.

It can be concluded that the concept of scaling all EMPFD variables in terms of energy and energy-rate is feasible, but the following issues must be addressed:

- If the (background) speed scale is calibrated in constant $\Delta \mathrm{V}_{\text {TRUE }}$ /inch, then the actual $\mathrm{V}_{\mathrm{CAS}}$ scale becomes nonlinear. The resulting $\mathrm{V}_{\text {CAS }}$ speed tape must be demonstrated to be acceptable.

- The scaling requirements dictate that, if a linear FPA scale and a linear speed scale in terms of true airspeed/inch are chosen, the vertical speed scale and altimeter scale need to have a dynamic range (variable scaling). Furthermore, equal $\Delta$ kinetic and $\Delta$ potential energy relative to a common reference cannot be represented by exactly the same bar lengths on the speed and altitude scales.

- The FPA information used in the center of the EMPFD, must be referenced to inertial space (the earth), to avoid a conflict with the display of the vertical speed, which is referenced to inertial space.

- The acceleration information, used for the acceleration indication bar and the computation of PFPA (thrust setting cue) in the center of the EMPFD, must be referenced in the low frequency range to true airspeed to provide correct thrust guidance cuing in windshear, but derived from inertial acceleration in the high frequency range to filter out atmospheric noise. However, this is in conflict with the definition of PFPA, as defined in (1a). This may result in an error in the PFPA indication used for thrust setting, in the presence of a significant horizontal and/or vertical wind, unless an appropriate correction is applied to the acceleration component of PFPA indication.

- It should also be noted that during climb or descent at constant calibrated airspeed, the true airspeed is changing. As a result a further correction is needed to the airmass-referenced acceleration, used for both the acceleration bar and the airmass acceleration component of the PFPA indication. These PFPA correction issues also apply to HUD designs.

- To visualize the distribution of the energy-rate as shown in Figures 9 to 11 requires the null reference position of the acceleration and vertical speed tapes to be aligned with the null reference of the FPA scale (the horizon line). The same is true for the null reference location of the speed tape and the altitude tape. This conflicts with a guideline from [3] where it is stated that 'moving scale air data displays should have their present value aligned with the center of the attitude display fixed airplane reference'.

- Given that the primary guidance cue is the FPA marker (velocity vector), there may be advantages to aligning the FPA marker with the display center, instead of aligning the aircraft attitude marker with the center of the display. This concept was explored by Steinmetz [18] and compared with an attitude aligned format. Steinmetz reports that 'overall, the pilots preferred the display format aligned with the velocity vector' and 'the flight and simulation tracking performance for glide-slope and localizer signals were excellent and 
would meet category II or III

requirements'.

A pilot-in-the-loop evaluation will be needed to fully assess the effects of the speed- and altitude energy scaling differences.

\section{Design Example}

In the previous section it was concluded that the concept is feasible. The next question that needs to be addressed is whether the concept is practical. Given the dependencies between the different scales, it may well be that scaling requirements in terms of the desired range and resolution for each of the scales cannot be met. The following design example addresses this question.

\section{Computing the Ranges of the Scales}

In the previous section it was pointed out that if a constant scaling for the pitch attitude and FPA is selected, the vertical speed scale and altimeter scale need to have a dynamic range.

As a first step, the desired visible range of the pitch scale is specified. In [2] it is recommended that ' With the aircraft symbol on the horizon line, the range of visible pitch attitude shall be at least $+25^{\circ}$ to $-15^{\circ}$, but not to exceed a total pitch range of $50^{\circ}$. In this example, the range is selected to $50^{\circ}$ (similar to most PFDs). For the display in Figure 13 , the scale goes from $-25^{\circ}$ to $+25^{\circ}$, hence the location of $\mathrm{FPA}_{\mathrm{MAX}} / 2$ is at $12.5^{\circ}$. For this situation (red line in Figure 13), $\mathrm{a}_{\mathrm{MAX}}$ is equal to $4.2 \mathrm{kts}$ true airspeed/sec.

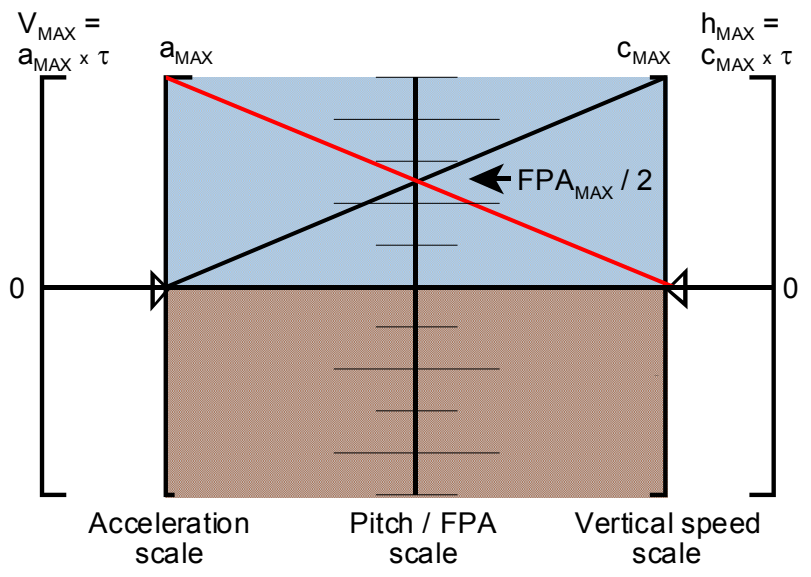

Figure 13. Scaling Dependencies

From this and the specification of the guidance time constant between energy and energy-rate, the range of the speed tape can be determined. In [2], the following is stated regarding the range on the speed tape: 'number of knots displayed $\min \pm 40$ knots, max \pm 65 knots'. With a time constant of 10 seconds (which is the typical value used to integrate acceleration into a speed trend vector, and also the value of the speed and altitude capture time constants used in TECS for automatic control), $\mathrm{V}_{\text {MAX }}$ becomes $42 \mathrm{kts}$ in terms of true airspeed. The full visible range of the speed tape then becomes 84 kts, already meeting the recommendation from [2]. In terms of calibrated airspeed, the range is increasingly larger with increasing altitude. Note that this is the range for the length of the tape that is equal to the height of the attitude indicator. In case the design requires a larger range, e.g. $100 \mathrm{kts}$, this can be achieved by extending the tape 8 kts up and $8 \mathrm{kts}$ down. The resulting range is comparable to today's PFDs.

The next step is the computation of the range of the vertical speed and altitude scale. As pointed out, this range depends on speed. For this example, a speed of $200 \mathrm{kts}$ is used. Consequently, the range will be proportional to the ratio of actual speed over $200 \mathrm{kts}$. For the situation of the black line in Figure $13, \mathrm{c}_{\mathrm{MAX}}$ is equal to $74.3 \mathrm{ft} / \mathrm{sec}$, which is approximately $4460 \mathrm{ft} / \mathrm{min}$. This is a bit less than the range of some contemporary vertical speed indicators, but similar to extending the speed tape, the designer can decide to extend the scale beyond the size of the attitude indicator to reach a value of $6000 \mathrm{ft} / \mathrm{min}$. In [2] it is recommended that 'Analog presentation beyond 3000 FPM is not required if supported by a digital readout' and 'At least 3000 FPM is required for TCAS II maneuvering. Proposed future logic changes may require up to 6000 FPM'. Furthermore, it must be noted that the range is dynamic and proportional to speed. Hence, for larger speeds, the range will also increase and for lower speeds the scale will provide an increased resolution.

The final scale that needs to be computed is the altitude tape. It is necessary to use the same scaling ratio of the altitude and vertical speed tapes as used for the scale ratio of the speed and acceleration tapes. In this example, 10 seconds yields a value of $h_{\text {MAX }}$ of 743 feet at a speed of 200 kts. As a result, the full visible range is equal to 1486 feet. In the evaluation of candidate formats 
for the 747-400, Konicke [1] reports that a format with an altitude range of $1400 \mathrm{ft}$ was considered to provide poor altitude sensitivity. The format with a range of $600 \mathrm{ft}$ provided adequate sensitivity but the range was found to be marginal. Konicke [1] does not report whether this problem occurred at a particular speed range. It was solved by increasing the vertical size (and hence the range) of the visible area of the altitude tape. Given these findings, there may be an issue regarding the altitude sensitivity at higher speeds. On the other hand, the presence of the digital altitude readout may compensate for this and the fact that at higher speeds more altitude preview is available may even turn out to be an advantage. Regarding the question about practicality, it can be concluded that the designer has sufficient freedom to achieve the desired range on all variables indicated on the EMPFD.

\section{Implementation}

In the previous sections it was concluded that the concept of the display is both feasible and practical. Hence, the next question is whether the display is suitable. This section provides examples to illustrate the potential suitability. In the next section, suggestions are provided for performance measures that could be obtained from data of pilotin-the-loop evaluations.

To utilize the advantages of the energy scaling for both manual control and monitoring of the automation, a number of parameters have been visualized using additional symbology. Figure 14 shows a dynamic situation with the most important symbology indicated.

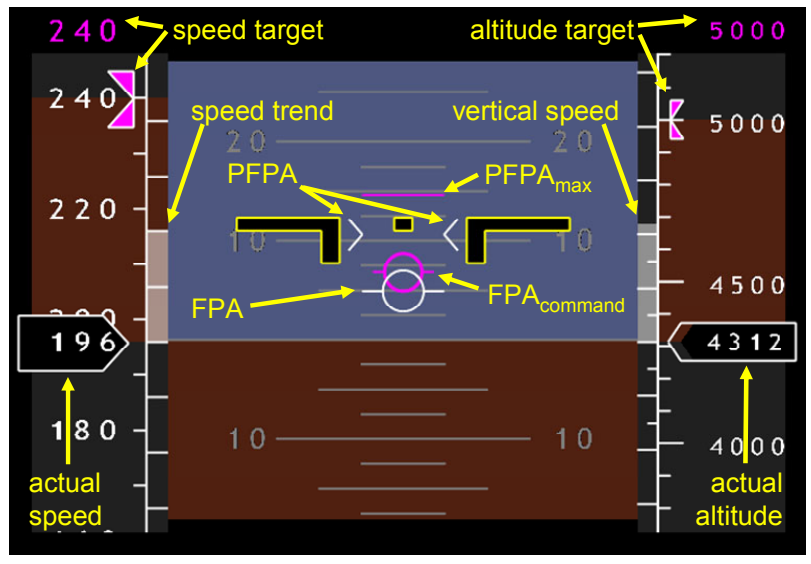

Figure 14. Explanation of Symbology
One of the non-standard indications is the marking of the tape between the target value and the actual value visualized by a brown shaded area. These markings correspond to the amount of kinetic (left) and potential (right) energy that the airplane has to gain or loose to reach the speed and altitude targets. On the inside of these markings the actual acceleration or deceleration and actual vertical speed are indicated. In the center of the display there are two distinct sets of symbols related to the FPA. There is a regular white FPA symbol with two symmetrical white carets for the acceleration cue, also called the PFPA marker. There is also a magenta FPA-Command symbol and a PFPA-Max symbol visualized by the magenta line. The concept behind these symbols was already discussed, except for the magenta FPA-Command and PFPA-Max symbols. In the manual control mode, the magenta FPA-Command symbol indicates the FPA command from the FBW computer, in effect a quickened FPA, generated by the pilot's pitch control input. In the automatic modes the magenta FPA-Command marker indicates the instantaneous FPA command. The PFPA-Max symbol represents the maximum PFPA available when the thrust is advanced to the forward limit.

In Figures 15-19 the behavior and operations will be illustrated. For the shown implementation a 46 degree pitch range is selected. This combined with a 10 second time constant for acceleration leads to a visible speed range of $80 \mathrm{kts}$ Calibrated (or Indicated) airspeed (Requirement from [3]). Figure 15 shows level flight at a speed of 140 kts and an altitude of $5000 \mathrm{ft}$.

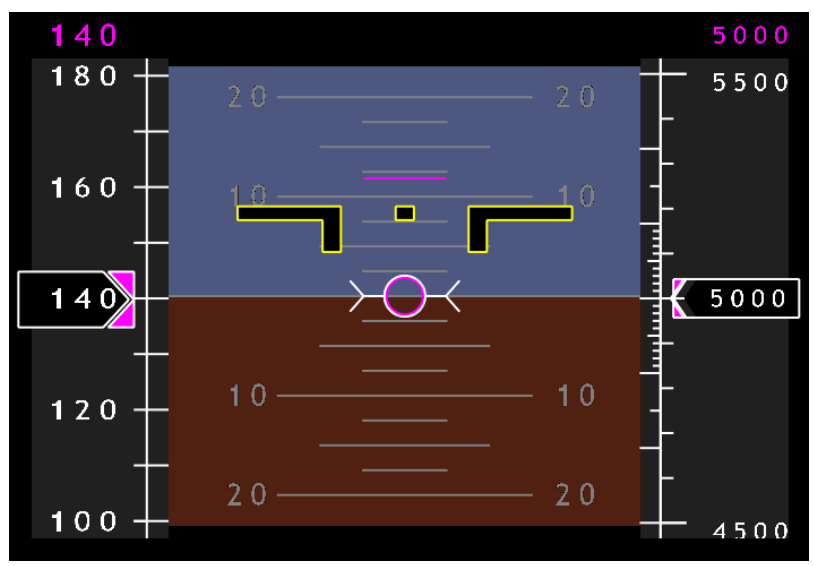

Figure 15. Straight and Level Flight 
In this situation the visible altitude range is about $1000 \mathrm{ft}$ and the vertical speed range is approximately $6000 \mathrm{ft} / \mathrm{min}$. The large vertical speed markers indicate $1000 \mathrm{ft} / \mathrm{min}$ increments. At low speeds an additional high resolution vertical speed scale is visible with $100 \mathrm{ft}$ increments for high accuracy vertical maneuvers. Figure 16 shows a climb in the automatic mode, from level flight to an altitude target of $10000 \mathrm{ft}$. The brown bar on the altitude tape shows the potential energy that the airplane must gain to arrive at the altitude target. The throttles have been advanced to climb power, as is indicated by the white PFPA symbol (carets) being lined up with the FPA-Max symbol. The white FPA symbol is lined up with the white PFPA marker and this indicates the current speed is being maintained, as is also indicated by the acceleration bar. The magenta FPA-Command symbol remains "parked" ahead of the actual FPA indication as long as the airplane is in a steady climb and will begin to move down at the time when the altitude command symbol has come down to a position that is level with the vertical speed indication. At that point the final altitude capture maneuver starts, the throttles will come off the forward limit and from that point on, the vertical speed indication will remain lined up with the altitude command target, as the altitude error decays to zero. The TECS automatic control modes will calculate a normal acceleration limited FPA command, control the throttles and the elevator such that for this case it will maintain the speed during the climb maneuver. In the FBW manual mode, the pilot uses his pitch control to put the magenta FPA command where he desires it by using his pitch control, after which the white FPA will follow with some delay. Both FPA and pitch attitude-centered displays have been implemented. In Figure 16, the FPA-centered implementation is visualized, which keeps the white FPA symbol in the center of the display.

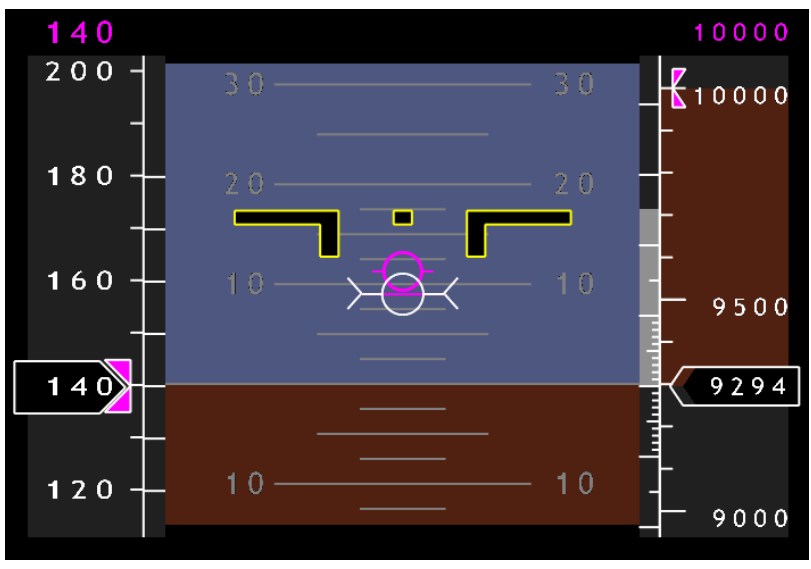

Figure 16. Performance Limited Steady State Climb

When comparing Figure 15 and Figure 16, it can be observed that the speed and altitude tapes have translated down, together with the horizon. The amount of translation depends on the alignment of the display (FPA centered or Pitch attitude centered) and the actual FPA. In case of the attitude-centered implementation the pitch attitude symbol would be in the center and the tapes would have translated down an additional 7 degrees representing the current angle of attack.

The advantage of the FPA centered display is that the vertical translation of the horizon and the speed and altitude reference points increases the display viewing range of altitude and speed in the direction of the Total Energy change and thus the thrust setting. This advances the time when the speed and altitude targets come into view, thereby enhancing the visualization of speed and/or altitude capture.

In Figure 17, with the automatic modes still engaged, the speed target was set to $250 \mathrm{kts}$, after capturing the altitude target. The FPA and FPAcommand symbols will stay on the horizon line. In this case there will be no translation of the tapes. The acceleration indication is moving up and back down in unison with the PFPA indication. While the thrust is at the forward limit, the PFPA symbol will remain pegged at the FPA-Max symbol. 


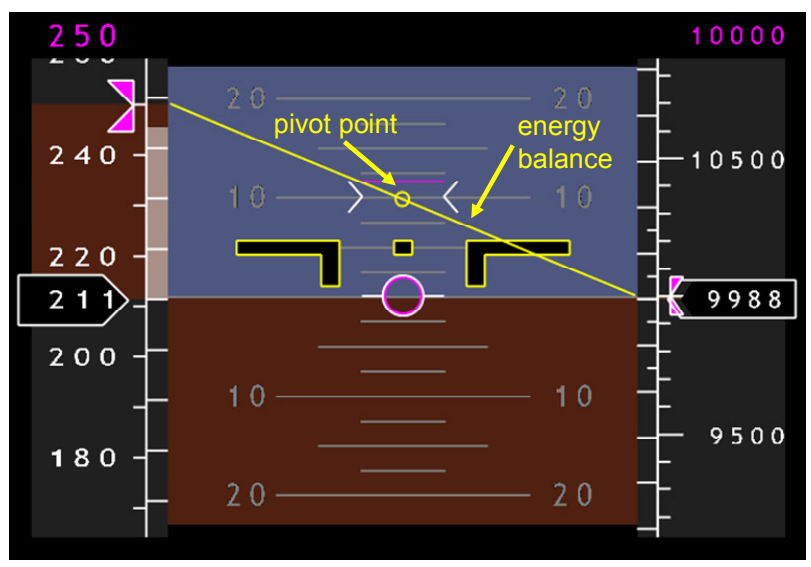

Figure 17. Capture of Speed Target

The TECS automatic modes also use a 10 second time constant for capturing speed and altitude targets. Thus, when the speed target symbol moves down to align with the acceleration indication bar, the automatic system will or the pilot should begin to reduce the thrust to keep the top of the acceleration bar aligned with the speed target, while the speed error decays to zero.

For illustration purposes the yellow energy balance line connecting the acceleration and vertical speed bars is visualized. With the pivot point for energy redistribution marked at the center of the PFPA symbology, it becomes obvious why the dual caret symbology has been chosen for the PFPA, instead of a regular single sided one. In the concept discussion it was explained that the pivot point is set with the throttle and the elevator is used to distribute the energy between kinetic and potential, indicated by the tilt angle of the yellow line showing the relative energy distribution.

For speed and/or altitude target changes in excess of the range of the indication scales, the target symbols will be parked at the top or bottom of the scale. To further help the pilot capture speed and altitude targets simultaneously using thrust and elevator control in the most efficient way, it is possible to add one more symbol to the display, placed at the center of the connecting line between the speed and altitude targets (not shown). This is the ideal guidance symbol for placing the PFPA symbol using the thrust setting, assuming the location is within the range of PFPA-Max and PFPA-Min (the achievable FPA when thrust is at idle and the acceleration is zero). If it falls outside, the pilot simply uses maximum or minimum thrust available, and controls the preferred speed or flight path target with the elevator, until this thrust guidance symbol comes within the range of PFPAMax and FPA-Min. At that point the pilot follows this thrust guidance symbol with the PFPA indication, using thrust adjustments. If both speed and altitude targets are far removed from the current speed and altitude, and opposite in direction, the thrust guidance indication will center on the horizon. This indicates that thrust can be left alone for a while, and that the pilot should first execute the energy exchange maneuver using the elevator, until the thrust guidance symbol begins to move.

Figure 18 illustrates a situation where we want to exchange a similar amount of kinetic energy towards potential energy. Both speed and altitude targets can be reached simultaneously by using the elevator to redistribute the surplus kinetic energy to fill the shortage in potential energy, without touching the throttle.

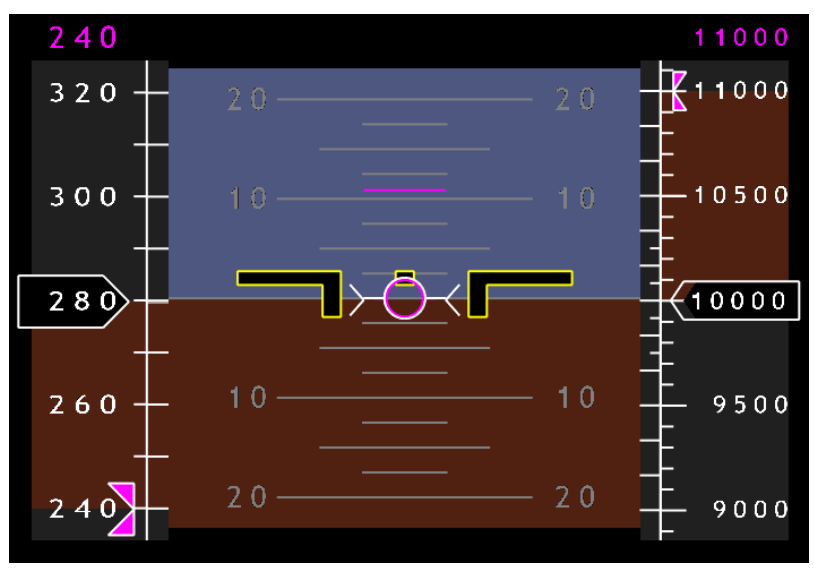

Figure 18. Start Condition Before Exchanging Equal Amount of Kinetic to Potential Energy

Figure 19 illustrates the progress during the capture. The elevator was used to convert about half of the kinetic energy surplus in Figure 18 to potential energy. The energy balance pivot point is still on the horizon, meaning that the throttle position is still the same. Both the kinetic and potential energy errors and the acceleration and vertical speed bars have equal and opposite lengths. That means, the speed and altitude targets will be reached at about the same time. 


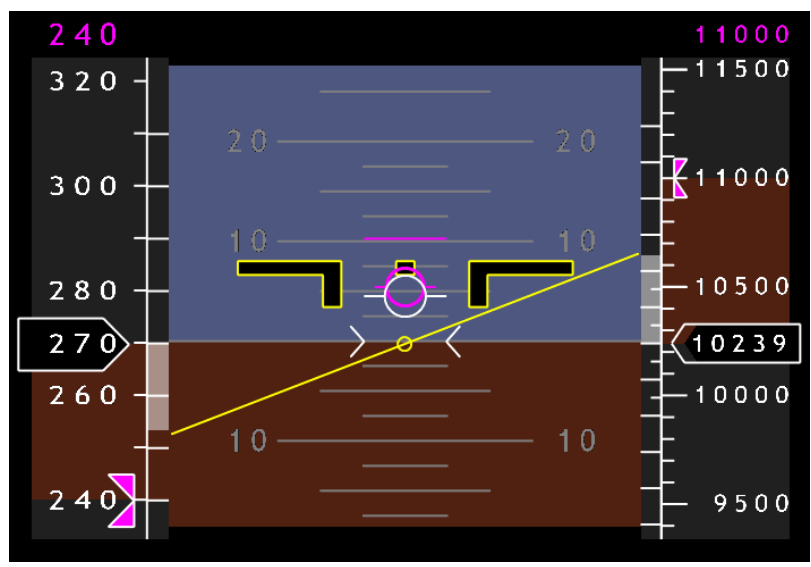

Figure 19. Capturing Targets with Minimal Use of Throttle

Using the EMPFD, energy efficient maneuvering with minimal undesirable up- and down throttle activity can be achieved. Since the TECS automatic control modes also work this way, the EMPFD seamlessly combines efficient energy management control guidance to the pilot with a monitoring capability for the operation of the TECS automatic control modes.

\section{Discussion and Future Research}

Replacement of today's PFD with an EMPFD on current transport and general aviation airplanes may prove a to be a long shot, even if the pilot in the loop simulation analyses shows well defined safety and performance benefits in terms of:

- Pilot awareness of energy state and airplane performance capabilities

- Improved control and energy efficiency (more MIMO control/less activity)

- Improved speed and altitude target tracking performance

- Faster ab-initio pilot training and acquisition of desired MIMO control skills

However, if the pilot in the loop simulation analyses reveals significant benefits in any of the above areas, then it may be attractive to further develop the EMPFD concept into a practical product, most likely to be applied on new airplane programs. The concept here described is not limited to conventional PFD applications. It may be equally suitable as an overlay on a synthetic vision display. Given a number of mishaps with UAVs, attributable to unawareness of dangerously low energy situations (e.g. causing a stall during the landing), remote piloting of unmanned aircraft may also benefit from an improved awareness of the energy state.

A further incentive for the application of this EMPFD concept will result when the automatic flight guidance and control systems also move to incorporate an energy-based control strategy, as the TECS design does. This would minimize the shift in mental model the pilot uses when going form manual control to automatic control, as is required with today's designs, yielding a true humancentered design [19].

In the concept discussed so far, the emphasis was on visualizing the relation between all relevant variables. As indicated, there are certain issues associated with this. A more conventional version, e.g. using equal scaling in energy in an attitudealigned format with a fixed location for the speedand altitude readouts, may already provide awareness of the energy state and in this way provide level 2 SA.

Future research will address the suitability and effectiveness in more detail. To be able to measure and quantify potential advantages, design of the evaluation tasks and criteria is essential. To compare the performance of an EMPFD with a conventional PFD, tasks need to comprise transitions in speed and altitude such as depicted in Figure 7. The evaluation measures should be indicative of the deviation from the optimal transition between the two states. Another reference could be the energy path taken by a SISO- and a TECS-based control system (as shown in Figures 2 and 4). Furthermore, the tasks should include the detection of dangerously low energy states. This can be achieved by having the pilot supervise the automation (using a conventional SISO-based system) and generating a reference path that in the end will cause the envelope protection to intervene due to a limited thrust situation. Measures should be indicative of the timeliness of the detection by the pilot, i.e. does the display provide sufficient level 3 SA to anticipate this situation.

\section{Summary and Conclusion}

A conventional PFD provides information that aids the pilot in using a SISO control strategy. The 
lack of explicit support for energy awareness requires experience from the pilot to determine the required amount of thrust and elevator control for acquiring and capturing speed and altitude targets. It has been illustrated how awareness of the energy state is needed to determine optimal control actions to transition from one state to another. An analysis of the process and the resulting information requirements led to the identification of the need for an equal scaling of the states in terms of energy error, energy-rate and energy distribution.

This paper has presented a conceptual display format that provides the pilot with an awareness of the energy state and guidance cues supporting a MIMO control strategy to capture and track a speed and flight path target simultaneously. Rather than striving for a completely new display format, a concept has been developed which conveys the required information using modifications and enhancements to a conventional PFD format.

It was also pointed out that such a PFD, in combination with an energy-based control system like TECS, provides the opportunity to move toward a flight deck design that is based on a common strategy for manual display-aided guidance and control, and automatic guidance and control with simple and effective crew monitoring capabilities.

\section{References}

[1] Konicke, M.L., 1988, 747-400 Flight Displays Development, AIAA 88-4439.

[2] Aerospace Recommended Practice ARP 4102/7, Electronic Displays, Society of Automotive Engineers, 1988.

[3] Advisory Circular 25-11 Transport Category Airplane Electronic Display Systems, U.S. DoT, FAA.

[4] Klopfstein, 1973, Rational Study of Aircraft Piloting.

[5] Robbins, R.E. and R.D. Simpson, 1991, Method and Apparatus for Aircraft Pitch and Thrust Axes Control, U.S. Patent 5,031,102.

[6] Soule, H.A., 1969, The Throttle Controls Speed, Right? Wrong, Astronautics \& Aeronautics, December 1969, pp. 14,15,81.
[7] Lambregts, A.A., 1996, Automatic Flight Controls Concepts and Methods, Netherlands Society of Aeronautical Engineers.

[8] Lambregts, A.A., 1983, Vertical Flight Path and Speed Control Autopilot Design Using Total Energy Principles, AIAA Paper 83-2239CP.

[9] Arntz, N., Goo, A.M.S., Murphy R.D., 1989, CONDOR for High Altitudes, Aerospace America, Feb. 1989, pp. 36-37.

[10] Lambregts, A.A., 1983, Integrated System Design for Flight and Propulsion Control Using Total Energy Principles, AIAA Paper 83-2561CP.

[11] Feynman, R.P., 1942, The Principle of Least Action in Quantum Mechanics, Doctoral thesis.

[12] Endsley, M.R., 1995, Towards a New Paradigm for Automation: Designing for Situation Awareness, Proceedings of the '95 IFAC MMS Conference, pp. 421-426, June 27-29, Cambridge, MA.

[13] McRuer, D.T., Weir, D.H., and Klein, R.H., 1971, A Pilot-Vehicle Systems Approach to Longitudinal Flight Director Design, Journal of Aircraft, Vol. 8, No. 11, pp. 890-897.

[14] Lambregts, A.A. and D.G. Cannon, 1979, Development of a Control Wheel Steering Mode and Suitable Displays that Reduce Pilot Workload and Improve Efficiency and Safety of Operation in the Terminal Area and in Windshear, AIAA Paper 79-1887CP.

[15] Lambregts, A.A., 1991, Aircraft Flight Path Angle Display System, U.S. Patent 5,016,177.

[16] Bray, R. S., and B.C. Scott, 1981, A Head-Up Display format for transport aircraft approach and landing, The 1980 Aircraft Safety and Operating Problems, 165-193.

[14] Newman, R.L., 1995, Head-Up Displays Designing the Way Ahead, Ashgate Publishing Company, ISBN 0291398111.

[18] Steinmetz, G.G., 1986, Development and Evaluation of an Airplane Electronic Display Format Aligned With the Inertial Velocity vector, NASA Technical Paper 2648, Langley Research Center, Hampton, VA. 
[19] Billings, C.E., 1991, Human-Centered Aircraft Automation: A Concept and Guidelines, NASA Technical Memorandum 103885. 27th Digital Avionics Systems Conference October 26-30, 2008 